\title{
MEAN FLOW EFFECTS IN MODEL EQUATIONS FOR FARADAY WAVES
}

\author{
Sten Rüdiger and José M. Vega \\ Physikalisches Institut, Universität Bayreuth, 95440 Bayreuth, Germany \\ $\dagger$ E.T.S.I. Aeronáuticos, Universidad Politécnica de Madrid. \\ Plaza Cardenal Cisneros, 3. 28040 Madrid, SPAIN
}

\begin{abstract}
We review model equations for parametric surface waves (Faraday waves) in the limit of small viscous dissipation. The equations account for two effects of viscosity, namely damping of the waves and slowly varying streaming and large scale flows (mean flow). Equations for the mean flow can be derived by a multiple scale analysis and are coupled to an order parameter equation describing the evolution of the surface waves. In addition, the equations incorporate a phenomenological damping term due to viscous dissipation. The nonlinear terms, which are undetermined by the derivation of the equation for the surface waves, are chosen so that the primary bifurcation is to a set of standing waves in the form of stripes. Results for the secondary instabilities of the primary waves are presented, including a weak amplification of both Eckhaus and Transverse Amplitude Modulation instabilities due to the mean flow, and a new longitudinal oscillatory instability which is absent without mean flow. Generation of mean flow due to dislocation defects in regular patterns is studied by numerical simulations.
\end{abstract}

\section{Introduction}

Parametrically driven surface waves, also known as Faraday waves, are created when a layer of an incompressible fluid is vibrated periodically in the direction normal to the free surface at rest [1]- [4]. Standing surface waves emerge above the primary instability of the planar surface forming a stationary pattern with a symmetry that depends on the fluid parameters and the frequency of the forcing [5]- [8]. While a description of the fluid with conservative equations suffices to explain the excitation of surface waves, consideration of viscosity is necessary to address further characteristics of the waves. A first question concerns the effect of damping on the saturation of amplitudes and on the selection of patterns. Milner [9] has shown how 
nonlinear terms from an expansion of the energy dissipation due to viscosity in the bulk of the layer mediate nonlinear wave interaction and lead to nonlinear terms in the amplitude equation and therefore to the saturation of amplitudes. Furthermore, the specific form of the coefficients of the nonlinear terms is dominated by triad resonant interactions which govern the selection of patterns [10]- [12].

A further important effect related to viscosity is the existence of slowly varying flows or mean flows. To date, most theories of parametric surface waves near onset have neglected such flows despite the observation [13] that their effect is of the same order as the standard cubic nonlinear and conservative terms which are usually retained. Thus weakly nonlinear corrections to surface waves and mean flows must be considered simultaneously [14][18]. A consistent derivation of mean flow equations for Faraday waves requires explicit consideration of special limits, in particular for the physical dimensions of the container. While the relevant coupled amplitude-mean flow equations have been already derived for horizontally one-dimensional waves [13], the extension to two-dimensional waves is not straightforward; a first step in this direction has been made by Vega et al. [19]. Two separate contributions to mean flow were found: An inviscid contribution originating from the slowly varying motion of the free surface, similar to the one appearing in classical Davey-Stewartson models [20], and a viscous one resulting from a slowly varying shear stress produced by time-averaged Reynolds stress in the the oscillatory boundary layer attached to the free surface or the lower plate. The latter describes diffusive or convective vorticity transport from the boundary layer into the bulk [21].

In this article we review the work on mean flow equations derived by a rigorous multiple scale analysis in the limit of weak viscous dissipation coupled to a phenomenological equation. We address the consequences of the mean flows in a laterally unbounded geometry, namely changes in the response of the base pattern of standing waves to perturbations (secondary instabilities) and the generation of mean flows by isolated defects. To simplify the analysis the cubic nonlinear terms of the phenomenological model were chosen such as to lead to a stripe pattern above onset instead of a square pattern which was experimentally observed in the limit of weak viscous dissipation. While it is easy to modify the functional form of the cubic term to produce square patterns, it is natural to first clarify the effect of mean flows on stripe patterns. It is found that the mean flow couples to perturbations of the stripe pattern in different strength depending on the type of perturbation. In general, the incorporation of mean flow is destabilizing 
with the strongest coupling occurring for longitudinal perturbations. Moreover, the mean flow generates a qualitatively new oscillatory longitudinal perturbation which for small nonlinear damping in the phenomenological equation renders all stripe patterns unstable resulting in time-dependent patterns at onset. By tuning the nonlinear damping to larger values one can generate a range of stable stripe patterns. In this latter situation we consider the effect of mean flows for irregularities in the pattern. Here the simplest case of a pair of dislocations in a pattern of perfect stripes is chosen. Using numerical simulations it is found that the flow generated by a dislocation is a large scale flow consisting of two vortices. In the parameter range considered the mean flow increases the velocity of the dislocation.

The article is organized as follows: In Section 2 we present the nondimensional equations. Section 3 justifies the phenomenological equation for a complex order parameter $\psi$ that describes the evolution of the surface waves. The derivation of this equation is based on the assumption of an irrotational flow in the layer. In Section 4 we sketch the derivation of the mean flow equations and describe the coupling of mean flow and $\psi$. We then present results of the calculation of secondary instabilities in Section 5. Section 6 contains first results on the numerical simulation of the coupled equations to calculate mean flow effects on patterns with dislocations.

\section{Basic Equations}

Let a fluid layer of unperturbed depth $d^{*}$ be supported by a horizontal plate that is vibrating vertically with an amplitude $a^{*}$ and a frequency $2 \omega^{*}$, where the superscript ${ }^{*}$ denotes dimensional quantities. To obtain nondimensional equations we introduce the characteristic time $1 / \omega^{*}$ and length $1 / k^{*}$, where the wavenumber $k^{*}$ is related to $\omega^{*}$ by the inviscid dispersion relation

$$
\omega^{* 2}=g^{*} k^{*}+\sigma^{*} k^{* 3} / \rho^{*},
$$

in terms of the gravitational acceleration $g^{*}$, the surface tension $\sigma^{*}$ and the density $\rho^{*}$, which are all assumed constant. Moreover, we assume that the wavelength $k^{*-1}$ is small compared with the depth of the container. The resulting dimensionless incompressibility and Navier-Stokes equations in a reference frame attached to the vibrating container are

$$
\begin{aligned}
& \boldsymbol{\nabla} \cdot \boldsymbol{u}+\partial_{z} w=0 \\
& \partial_{t} \boldsymbol{u}-w\left(\boldsymbol{\nabla} w-\partial_{z} \boldsymbol{u}\right)-\boldsymbol{u}^{\perp} \nabla \cdot \boldsymbol{u}^{\perp}=-\nabla p+\gamma\left(\boldsymbol{\nabla}^{2} \boldsymbol{u}+\partial_{z z}^{2} \boldsymbol{u}\right) / 2, \\
& \partial_{t} w+\boldsymbol{u} \cdot\left(\boldsymbol{\nabla} w-\partial_{z} \boldsymbol{u}\right)=-\partial_{z} p+\gamma\left(\nabla^{2} w+\partial_{z z}^{2} w\right) / 2
\end{aligned}
$$


in $-d<z<h(x, y, t)$, with the $z=0$ plane at the unperturbed free surface. The boundary conditions result from no slip at the supporting plate,

$$
\boldsymbol{u}=\mathbf{0}, \quad w=0 \quad \text { at } z=-d,
$$

and kinematic compatibility and equilibrium of tangential and normal stresses at the free surface,

$\partial_{t} h+\boldsymbol{u} \cdot \boldsymbol{\nabla} h=w$

$\partial_{z} \boldsymbol{u}+\boldsymbol{\nabla} w=\left(\boldsymbol{\nabla} \boldsymbol{u}+\boldsymbol{\nabla} \boldsymbol{u}^{\top}\right) \cdot \boldsymbol{\nabla} h-\left[2 \partial_{z} w-\left(\partial_{z} \boldsymbol{u}+\boldsymbol{\nabla} w\right) \cdot \boldsymbol{\nabla} h\right] \nabla h$,

$p-\left(|\boldsymbol{u}|^{2}+w^{2}\right) / 2-[4 a \sin 2 t+1-\Gamma] h+\Gamma \nabla \cdot\left[\nabla h /\left(1+|\nabla h|^{2}\right)^{1 / 2}\right]$

$=\gamma\left[\partial_{z} w-\left(\partial_{z} \boldsymbol{u}+\boldsymbol{\nabla} w\right) \cdot \boldsymbol{\nabla} h+\left(\boldsymbol{\nabla} h \cdot\left(\boldsymbol{\nabla u}+\boldsymbol{\nabla} \boldsymbol{u}^{\top}\right) / 2\right) \cdot \nabla h\right] /\left(1+|\nabla h|^{2}\right)$

at $z=h$. Here $\boldsymbol{u}=(u, v, 0)$ and $w$ are the horizontal and vertical velocity components, $\boldsymbol{\nabla}=\left(\partial_{x}, \partial_{y}, 0\right)$ denotes the horizontal gradient, the superscript $\perp$ over a horizontal vector denotes the result of rotating the vector $90^{\circ}$ counterclockwise, namely $u^{\perp}=(-v, u, 0)$, and the superscript $T$ over a tensor denotes its transpose; $p$ (=pressure $\left.+\left(|\boldsymbol{u}|^{2}+w^{2}\right) / 2+[1-\Gamma+4 a \sin (2 t)] z\right)$ is a conveniently modified pressure, and $h$ is the (vertical) free surface deflection. For simplicity we do not consider lateral walls, but impose periodic boundary conditions in the two horizontal directions.

In the nondimensional system the following parameters remain: the dimensionless viscosity $\gamma=2 \nu^{*} k^{* 2} / \omega^{*}$ (with $\nu^{*}=$ kinematic viscosity), the gravity-capillary contribution $\Gamma=\sigma^{*} k^{* 3} /\left(\rho^{*} \omega^{* 2}\right)$, the forcing amplitude $a=a^{*} k^{*}$, the container depth $d=d^{*} k^{*}$ and the aspect ratios $L_{1}$ and $L_{2}$. According to Eq. (1), $0 \leq \Gamma \leq 1$ where the extreme cases $\Gamma=0$ and 1 correspond to the purely gravitational and purely capillary limits respectively.

\section{Derivation of Model Equations}

Following similar considerations in other systems such as the RayleighBénard convection [22] a phenomenological model equation was derived that can efficiently be used in analytical and numerical work to describe the evolution of the surface waves. Here we will outline the derivation of the phenomenological model first introduced in Zhang and Viñals [23] (see Zakharov [24] and Crawford et al. [25] for the original use of the method). They assume an inviscid, incompressible, and irrotational fluid that is parametrically driven and add linear viscous damping in a phenomenological way. The governing equation are given by a Laplace equation for the velocity potential $\varphi$ and boundary conditions on $\varphi$ and the surface deflection 
$h(\mathbf{x}, t)$ at the free surface which correspond to the kinematic compatibility (Eq. (2)) and Bernoulli's equation at the free surface.

It is well known that this problem admits a Hamiltonian formulation with Hamiltonian $H$ where the canonically conjugate variables are given by $h(\mathbf{x}, t)$ and the velocity potential on the surface $\varphi^{s}(\mathbf{x}, t)=\varphi(\mathbf{x}, z=h(\mathbf{x}))$. Phenomenological damping can be introduced by considering a dissipation function $Q\left(h(\mathbf{x}, t), \varphi^{s}(\mathbf{x}, t)\right)$. The resulting canonical equations of motion are,

$$
\begin{aligned}
\partial_{t} h(\mathbf{x}, t) & =\frac{\delta H}{\delta \varphi^{s}(\mathbf{x}, t)}, \\
\partial_{t} \varphi^{s}(\mathbf{x}, t) & =-\frac{\delta H}{\delta h(\mathbf{x}, t)}+Q\left(h(\mathbf{x}, t), \varphi^{s}(\mathbf{x}, t)\right),
\end{aligned}
$$

where the functional $Q$ determines the rate of viscous dissipation in Eqs. (9)-(10) so that

$$
\frac{d H}{d t}-\frac{\partial H}{\partial t}=\int d \mathbf{x} Q\left(h(\mathbf{x}, t), \varphi^{s}(\mathbf{x}, t)\right) \partial_{t} h(\mathbf{x}, t) .
$$

If the fluid is of low viscosity and depth is large compared to wavelength, one can assume that energy dissipation is dominated by the potential flow in the bulk [26]. The functional $Q$ can then be determined by equating the rate of dissipation in Eq. (11) to the rate of energy dissipation due to potential flow,

$$
\int d \mathbf{x} Q\left(h(\mathbf{x}, t), \varphi^{s}(\mathbf{x}, t)\right) \partial_{t} h(\mathbf{x}, t)=-\frac{\gamma}{2} \int d \mathbf{x} \int_{\infty}^{h(\mathbf{x}, t)} d z \nabla^{2}(\nabla \varphi)^{2}
$$

This equation has been used to determine $Q$ order by order in an expansion in the surface wave steepness $[9,27]$. To the order relevant here, one finds,

$$
\hat{Q}(\mathbf{k}, t)=-2 \gamma k^{2} \hat{\varphi}^{s}(\mathbf{k}, t)+\text { nonlinear terms, }
$$

where $\hat{Q}(\mathbf{k}, t)$ is the Fourier transform of $Q$.

The next step in the derivation is the introduction of a complex order parameter field [24],

$$
b(\mathbf{k}, t)=\sqrt{\frac{\omega(k)}{2 k}} \hat{h}(\mathbf{k}, t)+i \sqrt{\frac{k}{2 \omega(k)}} \hat{\varphi}^{s}(\mathbf{k}, t),
$$

where $\hat{h}(\mathbf{k}, t)$ and $\hat{\varphi}^{s}(\mathbf{k}, t)$ are the two dimensional Fourier transforms of $h(\mathbf{x}, t)$ and $\varphi^{s}(\mathbf{x}, t)$ respectively, and $\omega(k)$ is the inviscid dispersion relation 
in the dimensionless system. In terms of this new variable, the Hamiltonian system $(9,10)$ can be written as,

$$
\partial_{t} b(\mathbf{k}, t)=-i \frac{\delta H}{\delta \bar{b}(-\mathbf{k}, t)}+i \sqrt{\frac{k}{2 \omega(k)}} \hat{Q}(\mathbf{k}, t) .
$$

Equation (15) can then be expanded in a power series of $b$ where one retains only terms linear in $b$, as nonlinear terms will be added phenomenologically.

Near onset only amplitudes with wavenumber close to the critical wavenumber are excited, with frequency close to the resonant frequency $\omega=1$. This facilitates a conventional multiple scale expansion near onset, chosen such that the rotational invariance of the original governing equations is preserved. Expanding

$$
b(\mathbf{k}, t)=\varepsilon B\left(\mathbf{k}, T_{1}, T_{2}\right) e^{-i t}+\mathcal{O}\left(\varepsilon^{2}\right)
$$

with $T_{1}=\varepsilon t$ and $T_{2}=\varepsilon^{2} t$ slow time scales corresponding to the time scale of translation of a wave packet and of change in the modulation of the wave packet, respectively, one can use the solvability conditions at orders $\mathcal{O}\left(\varepsilon^{2}\right)$ and $\mathcal{O}\left(\varepsilon^{3}\right)$ to obtain an equation for the evolution of $B$ on the slow time scales. One finally defines a complex order parameter field $\psi(\mathbf{x}, t)$ as the inverse Fourier transform of $B(\mathbf{k})$, and finds,

$$
\partial_{t} \psi=-\gamma \psi+\mathrm{i} f \bar{\psi}+3 \mathrm{i}\left(1+\nabla^{2}\right) \psi / 4+(\mathrm{i}-\gamma \alpha)|\psi|^{2} \psi,
$$

where $f$ is a parameter proportional to the amplitude $a$ of the vibrations. Three simplifications are necessary to obtain this equation. First, the nonlinear functional does not have a closed form representation in real space. As has been done in other systems (cf. Rayleigh-Bénard convection [22]), one introduces phenomenological functional forms for this term thereby choosing the symmetry of the bifurcating pattern at onset artificially. In addition, in the case of Faraday waves the issue of the origin of nonlinear damping and saturation of the waves is sidestepped $[12,28]$. In the simplest possible case, the coefficient of the nonlinear term is approximated by an imaginary constant which has already been rescaled to $i$ in Eq. (17). Second, it is also known that linear damping is not sufficient to produce wave saturation in this system [9]. Therefore a phenomenological nonlinear damping coefficient $\alpha \gamma$ is used, where $\alpha$ is a constant assumed to be of order 1.

The positive sign of the imaginary part of the nonlinear coefficient $(i-\gamma \alpha)$ in Eq. (17) is chosen to represent capillary waves [29]. In the opposite limit of gravity waves, the imaginary part of this coefficient has to 
be negative. We finally note that as a third simplification a further linear term $i\left(1+\nabla^{2}\right)^{2} \psi$ has been eliminated in Eq. (17) as this term together with $i\left(1+\nabla^{2}\right) \psi$ leads to two different wavenumbers becoming critical at threshold, which is an unwanted feature.

\section{Mean Flow Equations}

We now want to discuss the mean flow equations that have been derived by Vega et al. [19] using a multi-scale analysis in both (horizontal) space and time. The approximation requires that (i) the aspect ratio of the container be large, (ii) the surface waves are weakly damped and (iii) exhibit a small wavelength compared to the container's depth and (iv) a small steepness, which in turn require that $L \gg 1, \quad d \gg 1, \quad \gamma \ll 1, \quad|\nabla h| \ll 1, \quad a \ll 1$, where $L \leq \min \left\{L_{1}, L_{2}\right\}$ is a slow horizontal scale in which spatial modulations occur. Furthermore, it is assumed that $d$ is logarithmically large compared to the remaining small parameters (namely $\gamma, a$ and $L^{-1}$ ) and $d$ is treated as a $O(1)$ parameter. The limit is therefore chosen for simplicity such that

$$
\gamma^{2} \ll \mathrm{e}^{-d} \ll L^{-1} \sim \gamma \sim a \sim \varepsilon^{2},
$$

where $\varepsilon$ is a measure of the surface wave amplitude.

Here we want to sketch the important steps of the method used by Vega et al., and begin with a decomposition of the flow variables and the free surface deflection into oscillatory and time-averaged parts. They are associated with the surface waves and the mean flow (denoted hereinafter with the superscripts $o$ and $m$ ), respectively, as

$$
(\boldsymbol{u}, w, p, h)=\varepsilon\left(\boldsymbol{u}^{o}, w^{o}, p^{o}, h^{o}\right)+\varepsilon^{2}\left(\boldsymbol{u}^{m}, w^{m}, p^{m}, h^{m}\right),
$$

where $\varepsilon$ serves as the small parameter. The oscillatory flow variables associated with the surface waves are required to be such that

$$
\left\langle\boldsymbol{u}^{o}\right\rangle^{t s}=\mathbf{0}, \quad\left\langle w^{o}\right\rangle^{t s}=\left\langle p^{o}\right\rangle^{t s}=\left\langle h^{o}\right\rangle^{t s}=0,
$$

with $\langle\cdot\rangle^{t s}$ standing for the time average in the basic oscillating period

$$
\langle\psi\rangle^{t s}=(2 \pi)^{-1} \int_{t}^{t+2 \pi} \psi d t .
$$

The variables associated with the mean flow are required to depend weakly on time.

An intermediate step in this analysis is a set of equations for the mean flow and the oscillatory part, with the latter, for the current purposes, ultimately replaced by the phenomenological model equation obtained above. 
The intermediate equations for the surface waves are derived by a decomposition using $\varepsilon$, finding at first order an irrotational oscillatory flow in the bulk:

$$
\begin{gathered}
\boldsymbol{u}^{\circ}=\nabla \varphi+O\left(\varepsilon^{2}\right), \\
w^{o}=\partial_{z} \varphi+O\left(\varepsilon^{2}\right), \quad p=-\partial_{t} \varphi .
\end{gathered}
$$

As already mentioned in the introduction the mean flow will be forced in two basic ways. First we must take into account the oscillatory boundary layer attached to the free surface, which provides a slowly varying shear stress at the edge of this layer, that must be imposed as a boundary condition for the mean flow in the bulk. This forcing mechanism was first uncovered by Longuet-Higgins [21], who obtained an explicit expression for the forcing shear stress produced by general boundary layers in 2-D. The counterpart of this expression in 3-D has been only obtained recently by Nicolás and Vega [30]. The general formulae for the shear stress yields

$$
\begin{aligned}
\partial_{z} u^{m}+\nabla w^{m}= & 2\left\langle\nabla\left(\nabla \cdot\left(h^{o} \nabla \varphi\right)\right)\right. \\
& \left.+\left(\nabla h^{o} \cdot \nabla\right) \nabla \varphi+\left(\nabla^{2} \varphi\right) \nabla h^{o}\right\rangle^{t s} \quad \text { at } z=0
\end{aligned}
$$

where only the leading order contribution as $\gamma \rightarrow 0$ and $\varepsilon \rightarrow 0$ is retained. The boundary layer attached to the free surface has no effect on the other two boundary conditions at the unperturbed free surface, which are obtained from Eqs. $(8)$ and $(2,6,22)$ to be

$$
\begin{aligned}
& p^{m}-(1-\Gamma) h^{m}+\Gamma \nabla^{2} h^{m}=\left\langle h^{o} \partial_{t z}^{2} \varphi+\left(|\nabla \varphi|^{2}+\left|\partial_{z} \varphi\right|^{2}\right) / 2\right\rangle^{t s} \\
& \text { and } \partial_{t} h^{m}+\nabla \cdot\left(\int_{-d}^{0} u^{m} d z\right)=-\nabla \cdot\left(\left\langle h^{o} \nabla \varphi\right\rangle^{t s}\right) \quad \text { at } z=0,
\end{aligned}
$$

where we are only taking into account the leading order terms. We are neglecting the effect of a second oscillatory boundary layer attached to the lower plate because its effect is quite small (the oscillatory flow is weak near the lower plate as $\mathrm{e}^{-d}$ is small, Eq. (18)). These boundary conditions show that mean flow is forced by surface waves in two ways. The terms on the right hand sides of Eqs. (25) and (26) provide an inviscid forcing mechanism that drives an inviscid mean flow, like that appearing in the Davey-Stewartson model [20]. The right hand side of Eq. (24) instead produces a forcing shear stress that generates a viscous mean flow, which is absent in the usual inviscid and nearly inviscid theories of Faraday waves. Note that this forcing stress is generically non zero and independent of viscosity at leading order, a fact that is well known but somewhat surprising 
because this effect is due to the oscillatory boundary layer, and is absent in the strictly inviscid case [16].

The mean flow can be decomposed into its inviscid and viscous parts, as has been done in a number of studies $[13,17,18]$. Alternatively, a decomposition of the mean flow variables into a short wave component (oscillatory in the horizontal direction) and a long wave component (slowly varying in the horizontal direction) is a convenient choice. Their evolution has been derived by Vega et al. and is given by ${ }^{a}$

$$
\begin{aligned}
0 & =\nabla \cdot U^{m o}, \\
\partial_{t} U^{m o} & =-\varepsilon^{2} \nabla Q^{m o}+\gamma\left(\nabla^{2} U^{m o}-\frac{\beta_{1}^{4} \pi^{2}}{16} U^{m o}\right) / 2+\beta_{1} \gamma\left\langle\mathbf{N}^{v i s}\right\rangle^{h o} \\
\partial_{t} U^{m s} & =-\nabla Q^{m s}-\gamma \frac{\beta_{1}^{4} \pi^{2}}{32} U^{m s}+\beta_{1} \gamma\left\langle\mathbf{N}^{v i s}\right\rangle^{h s}, \\
4 Q^{m s} & =\beta_{1} \pi(1-\Gamma) h^{m s}, \\
\partial_{t} h^{m s} & =-\frac{4}{\beta_{1} \pi} \nabla \cdot U^{m s}-\nabla \cdot\left(\mathrm{i}\langle\psi \nabla \bar{\psi}\rangle^{h s}+c . c .\right),
\end{aligned}
$$

where $\mathbf{N}^{v i s}=\mathrm{i}(\nabla \psi \cdot \nabla) \nabla \bar{\psi}+\mathrm{i}\left(\nabla^{2} \bar{\psi}\right) \nabla \psi+$ c.c. denotes the viscous forcing terms and $\langle\cdot\rangle^{h s}$ and $\langle\cdot\rangle^{h o}$ project it onto its horizontal average in the short spatial scales and its short wave component. For a rigorous definition of these projections confer [19], where further details of the derivation can be found. Here we want to mention the following simplifications that are crucial to the method:

- Convective terms in the mean flow equations have been neglected thereby linearizing the mean flow equations. Since for standing waves the associated mean flow is unforced it identically vanishes at large times. Thus this approximation is exact for the linear stability analysis described below. Furthermore, one expects that the neglected convective terms do not play a significant qualitative role in subsequent bifurcated branches, at least near threshold.

- A single mode approximation for the $z$ dependence of the mean flow variables as $g(z)$ is introduced. This function is arbitrary and can be selected to yield the best approximation to the vertical velocity profiles. A reasonable choice is

$$
g(z)=\sqrt{2 / d} \sin [\pi(z+d) /(2 d)] .
$$

\footnotetext{
a The coefficient $\varepsilon^{2}$ in (28) disappears when time is scaled according to the slow time of the mean flow. This has not been done here for convenience.
} 
This function also determines the parameter $\beta_{1}$ in Eqs. (28-31) in terms of the depth $d$ of the layer:

$$
\beta_{1}=g(0)=\sqrt{2 / d}
$$

Finally we replace the complicated equations for the evolution of the oscillatory flow with the model equation (17) given above. To account for the effects of the mean flow on the surface waves we add a convective term $-\boldsymbol{u} \cdot \nabla \psi$ to the right hand side, where $\boldsymbol{u}$ is given by the two parts of the mean flow:

$\partial_{t} \psi=-\gamma \psi+\mathrm{i} f \bar{\psi}+3 \mathrm{i}\left(1+\nabla^{2}\right) \psi / 4+(\mathrm{i}-\gamma \alpha)|\psi|^{2} \psi-\beta_{1}\left(\boldsymbol{U}^{m o}+\boldsymbol{U}^{m s}\right) \cdot \nabla \psi$.

The coefficient $\beta_{1}$ originates from a similar coefficient in the corresponding term in the equation for the surface waves as derived in Vega et al.. Note that there is no dependence on $h^{m o}$ and $h^{m s}$ included, because this is beyond the scope of this phenomenological model.

\section{Bifurcations of Periodic Solutions}

We review in this section results on the instabilities of regular solutions of the model equations defined by the coupled Eqs. (27-31) and (34). The primary stability from the plane surface to standing waves is a linear stability problem in terms of perturbations in the form of spatially periodic patterns. As can be demonstrated from the form of the forcing terms in Eqs. (28-31) regular stripe patterns do not result in a mean flow, so that the primary instability is completely determined by Eq. (17). As has been described by Zhang and Viñals [23] the trivial solution $\psi=0$ becomes linearly unstable against a spatially periodic perturbation of $\psi$ of wave number $q$ for $\mu>\mu_{c}(q)=\sqrt{1+\left[3\left(1-q^{2}\right) / 4 \gamma\right]^{2}}-1$, the neutral stability curve; $\mu$ is the control parameter defined as $\mu=(f-\gamma) / \gamma$. The critical mode with $q=1$ becomes therefore unstable at $\mu=0$.

For small $\mu>0$ stationary and spatially periodic solutions exist that can be approximated by a single Fourier mode $\psi_{q}(x)=\alpha_{q} \cos (q x) \exp \left(\mathrm{i} \Theta_{q}\right)$ with

$$
\alpha_{q}^{2}=\frac{q^{2}-1-\frac{4}{3} \alpha \gamma^{2} \pm \frac{1}{3} \sqrt{16 f^{2}\left(1+\alpha^{2} \gamma^{2}\right)-\left(4 \gamma+3 \alpha \gamma\left(q^{2}-1\right)\right)^{2}}}{1+(\alpha \gamma)^{2}},
$$

where the \pm sign stands for $\operatorname{sign}\left(1-q^{2}+4 \alpha \gamma^{2} / 3\right)$, and $\Theta_{q}$ satisfies $\sin 2 \Theta_{q}=$ $\left(1+3 \alpha \alpha_{q}^{2} / 4\right) \gamma / f, \cos 2 \Theta_{q}=\frac{3}{4}\left(q^{2}-1-\alpha_{q}^{2}\right) / f$. Note that the bifurcation at threshold is subcritical if $q^{2}>1+3 \alpha \gamma^{2} / 4 \simeq 1$ (recall that $\gamma$ is small) and 
supercritical otherwise (a subcritical bifurcation for $q>1$ as $\gamma \rightarrow 0$ has also been found in a direct numerical simulation by Chen and Wu [31].) As mentioned above, this solution for the order parameter leads to vanishing driving terms in the mean flow Eqs. (28)-(31); hence all mean flow variables remain zero for the basic, periodic solution.

The criterion for linear instability yields a range of non-trivial solutions for every $\mu>0$. These solutions are possibly unstable and their linear stability (secondary stability) can be calculated by introducing small perturbations. Vega et al. [19] have studied the secondary stability using perturbations of general form. In order to address the stability of the primary stationary solution (35) against general longitudinal perturbations, they introduced

$$
\begin{aligned}
\psi= & A_{0}\left[\exp (\mathrm{i} q x)+\exp (-\mathrm{i} q x)+a^{++} \exp (\mathrm{i}(q+k) x)+a^{+-} \exp (\mathrm{i}(q-k) x)\right. \\
& \left.+a^{-+} \exp (\mathrm{i}(k-q) x)+a^{--} \exp (-\mathrm{i}(q+k) x)\right]
\end{aligned}
$$

(where $A_{0}=\frac{1}{2} \alpha_{q} \exp \left(\mathrm{i} \Theta_{q}\right)$ ), together with the corresponding perturbations of the mean flow variables

$$
U_{x}^{m s}=u^{+} \exp (\mathrm{i} k x)+c . c ., h^{m s}=c^{+} \exp (\mathrm{i} k x)+c . c .,
$$

and

$$
Q^{m s}=d^{+} \exp (\mathrm{i} k x)+\text { c.c. }
$$

where $U^{m o}=0$ as seen from the incompressibility condition (27) which requires that $U_{x}^{m o}=0$. A system of six first order differential equations for the perturbation amplitudes $a^{ \pm \pm}, u^{+}$, and $c^{+}$is derived by inserting the ansatz into Eqs. $(29-31,34)$. The linearization around the basic periodic solution is therefore given by a matrix $A(q, k, \varepsilon, \ldots)$ and the basic solution becomes unstable if the real part of an eigenvalue of $A$ becomes positive. Two relevant branches of eigenvalues appear in the vicinity of $k=0$. One is associated with the broken translational symmetry of the basic state $\psi_{q}(x)$, generating a mode which becomes marginal at $k=0$. The other branch is of hydrodynamic nature and corresponds to weakly damped uniform flows away from the quiescent state, with a damping rate of $\gamma \beta_{1}^{4} \pi^{2} / 32$ (Eq. (29)). The coupling of the two modes generates a steady long wavelength instability (modified Eckhaus) and a finite wavenumber oscillatory instability described below.

The stability of periodic solutions against transverse amplitude and phase perturbations can be studied similarly. We first note that given that $\partial_{y} \psi_{q}=0$ in the basic state with zero mean flow, terms involving the $y$ 
components of the mean flow will be of second order in the amplitudes of the perturbation and hence only the components $U_{x}^{m o}$ and $U_{x}^{m s}$ need to be perturbed. Here, in contrast to the case of a longitudinal perturbation, both short and long wavelength components of the mean flow need to be included. However, the equations for $U_{x}^{m o}$ and $U_{x}^{m s}$ decouple at linear order and can be analyzed separately.

Considering the short wavelength component of the mean flow velocity $U_{x}^{m o}$, and introducing the following perturbation for the order parameter,

$$
\begin{aligned}
\psi= & A_{0}\left[\exp (\mathrm{i} q x)+\exp (-\mathrm{i} q x)+a^{++} \exp (\mathrm{i}(q x+k y))\right. \\
& +a^{+-} \exp (\mathrm{i}(q x-k y))+a^{-+} \exp (\mathrm{i}(-q x+k y)) \\
& \left.+a^{--} \exp (-\mathrm{i}(q x+k y))\right], \\
U_{x}^{m o}=v^{++} & \exp (\mathrm{i}(2 q x+k y))+v^{+-} \exp (\mathrm{i}(2 q x-k y))+\text { c.c. },
\end{aligned}
$$

and

$$
Q^{m o}=p^{++} \exp (\mathrm{i}(2 q x+k y))+p^{+-} \exp (\mathrm{i}(2 q x-k y))+\text { c.c. },
$$

one can derive a linear system of equations for the perturbation amplitudes $a^{+ \pm}, a^{- \pm}$, and $v^{+ \pm}$. Suitable combinations of these modulations lead to the generalizations of the well-known transverse perturbations for Faraday patterns. A transverse amplitude modulation (TAM) is defined by the linear combinations $b_{1}=a^{++}+a^{+-}+a^{-+}+a^{--}$and $v_{1}=\operatorname{Im}\left(v^{++}+v^{+-}\right)$, whereas a transverse phase modulation (zig-zag) is given by $b_{2}=a^{++}-a^{+-}-a^{-+}+$ $a^{--}$and $v_{2}=\operatorname{Im}\left(v^{++}-v^{+-}\right)$.

A similar ansatz has been used for the long wavelength component of the mean flow with perturbations of the form $U_{x}^{m s}=u^{+} \exp (\mathrm{i} k y)+$ c.c., $h^{m s}=$ $c^{+} \exp (\mathrm{i} k y)+$ c.c., and $Q^{m s}=d^{+} \exp (\mathrm{i} k y)+$ c.c.. The order parameter is again given by Eq. (40).

From the eigenvalue equation of each of the resulting systems Vega et al. have demonstrated that modifications to the eigenvalues due to the mean flow are small compared to the case of longitudinal perturbations. Only through the coupling of TAM perturbations with the short wavelength component of the mean flow, the mean flow has a significant modification of the eigenvalues been found. However, its effect on the location of stability curves has been found to be small compared to the longitudinal case.

The numerically obtained results can be summarized in a $q-\mu$ diagram containing the different stability curves that together define the region of existence of stable periodic solutions. Figure 1 shows the various stability boundaries for the special cases of $\beta_{1}=0$ (no mean flow) and $\beta_{1}=0.5$. 
The other parameter values are $\gamma=0.1, \alpha=0.5$, and $\Gamma=0.8$. Except for $\alpha$, these values correspond approximately to those for the low-viscosity experiments described by Kudrolli and Gollub [5]. For instance, typical experimental values of $\beta_{1}=\sqrt{\pi / d}$ (where $d$ is the dimensionless height of the layer) are between 0.5 and 1.2. Figure 1 includes the neutral stability curve of the basic periodic solution and, since the primary bifurcation is subcritical for $q>1$, we have also included the saddle node curve where the periodic solution bifurcates. The case $\beta_{1}=0$ is shown as a reference, and it agrees with the results of Zhang and Viñals [23].

Basic solutions that are stable against all steady perturbations considered here (Eckhaus, TAM, and zig-zag) exist in a small region close to threshold at $\mu=0$ between the TAM and zig-zag lines. Periodic solutions are stable against transverse perturbations below the dashed-dotted line in the figure (zig-zag, denoted "Z"), and above the dashed line (TAM). Eckhaus perturbations have negative growth rate below the dotted line. We observe that with increasing $\beta_{1}$ both Eckhaus and TAM curves are shifted so that larger regions in the $(\mu, q)$ space become destabilized with respect to Eckhaus or TAM perturbations. As discussed above, the zig-zag line is not affected by the mean flow.

The picture just described changes dramatically if one allows for oscillatory longitudinal perturbations. The oscillatory Eckhaus perturbation leads to an instability provided that $\beta_{1} \neq 0$. This finding is consistent with the appearance of an oscillatory instability in the horizontally 1-D, large aspect ratio Faraday system [14], which is also absent when the effect of the mean flow is ignored. The oscillatory nature of the instability is apparent from the non-zero imaginary part of the eigenvalue $\sigma$ at the critical point in which $\operatorname{Re}(\sigma)=0$. Furthermore, the instability occurs at small but finite wavenumber $k$, a fact that has been confirmed by calculating the wave number with largest growth rate both slightly above and below the instability threshold. As mentioned above, the origin of the oscillatory instability can be traced back to the two eigenvalue branches that have small real parts for $k \rightarrow 0$. As $k$ increases, the two real eigenvalue branches merge leading to a complex conjugated pair and therefore to the oscillatory nature of the instability at finite $k$.

Results concerning the location of the oscillatory instability boundary as a function of $q$ for two values of $\beta_{1}$ are shown in Figure 2. Stable regions are located to the right of the plotted curves. As expected, the stability boundary moves toward the steady Eckhaus line with decreasing $\beta_{1}$, presumably merging with it for $\beta_{1} \rightarrow 0$. Note that for $\beta_{1}=0.5$ the unstable 

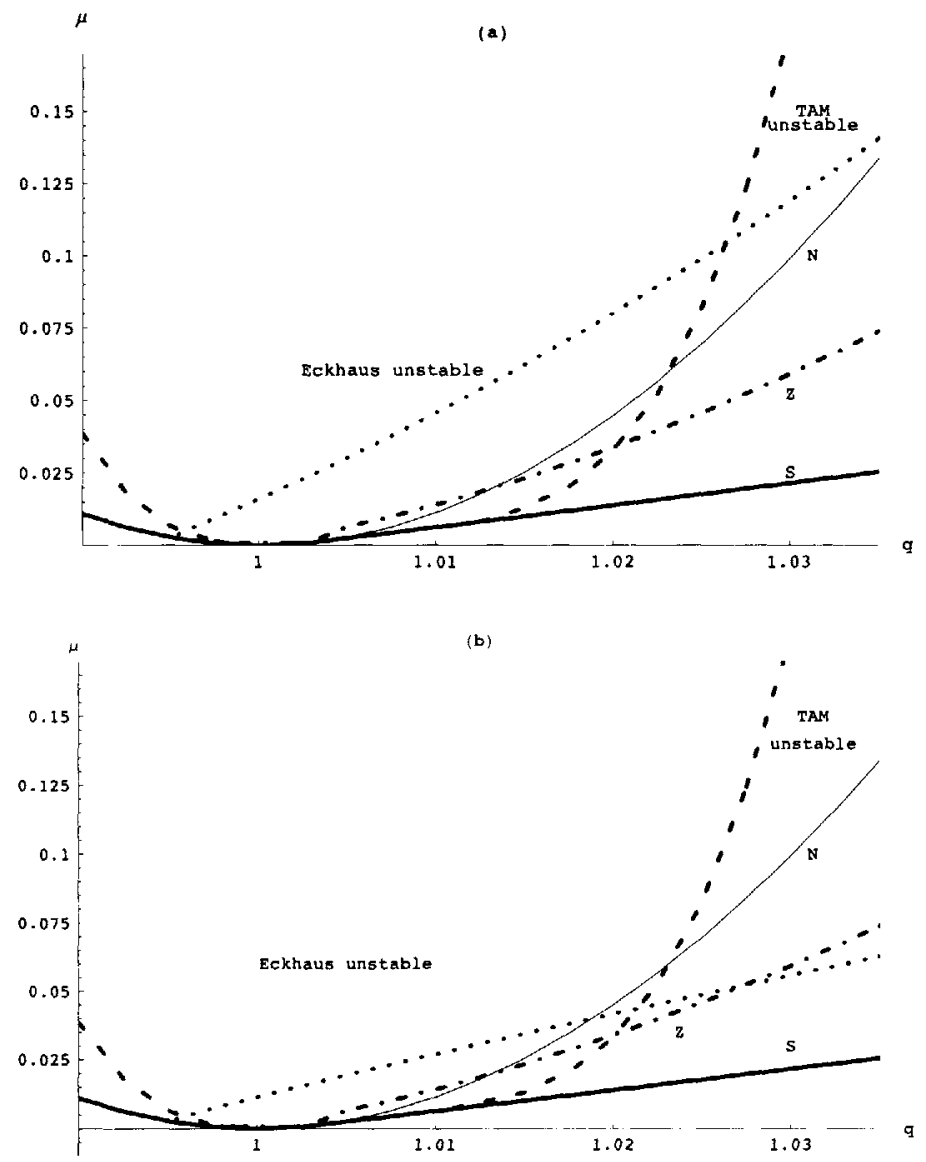

Fig. 1. Bifurcation diagram for (a) the order parameter model without mean flow, and (b) with $\beta_{1}=0.5$. Other values of the parameters are $\gamma=0.1, \alpha=0.5$, and $\Gamma=0.8$. The following stability lines are plotted: Eckhaus (dotted), TAM (dashed), zig-zag (dashdotted), neutral stability curve of the primary instability (solid line denoted by $N$ ), and a saddle node bifurcation (thick solid line marked $S$ ). Only the left branch of the Eckhaus line is shown emanating from $(q=1, \mu=0)$. The region of stability of the basic solution against an Eckhaus instability is the region below the dotted line. Comparison of (a) and (b) shows that the mean flow decreases the regions of stability against both Eckhaus instability and transverse amplitude modulation.

region covers most of the region of existence of the basic states except for a narrow stripe close to the saddle node bifurcation. 


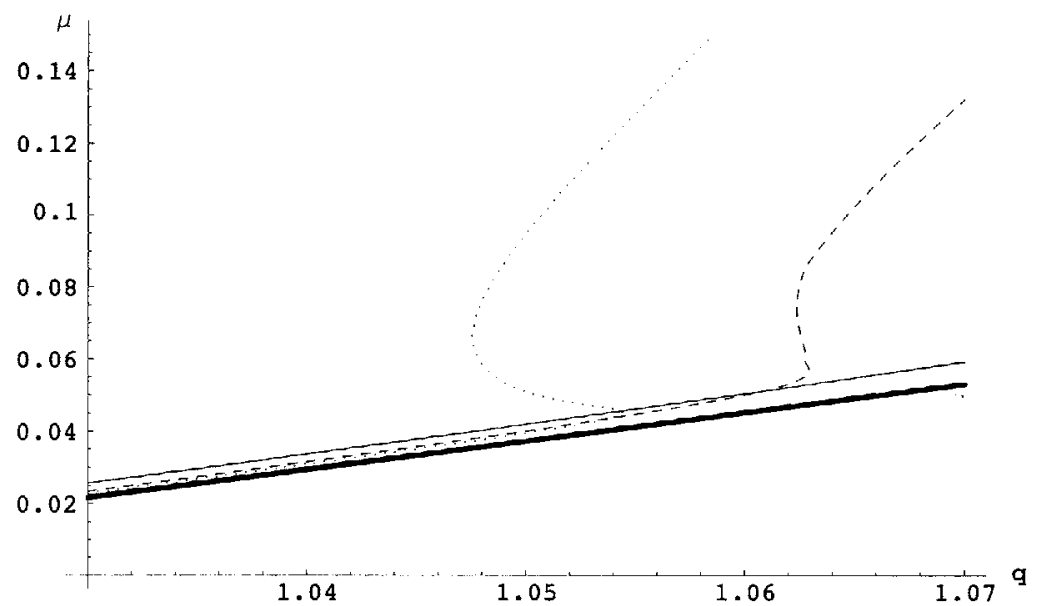

Fig. 2. Stability boundaries of the oscillatory instability for three values of $\beta_{1}: 0.05$ (dotted line), 0.2 (dashed line), and 0.5 (solid line). The thick solid line indicates the saddle node. Periodic solutions are unstable to the left of the curves.

\section{Mean Flow Generated by Defects}

In the following we will describe numerical simulations that have been performed to estimate the influence of the mean flow on the dynamical evolution of real patterns. Here we will consider the most simple configuration, a sample that contains two isolated dislocations. In general, the presence of a dislocation splits the sample into two regions which possess different wave numbers. If these two wave numbers are taken to be stable against secondary instabilities (according to the stability analysis described above) we can exclude that the defects are dynamically driven by a global instability. However, typically defects are found in motion which consists of two components: a climbing motion along the stripes and a gliding motion parallel to the wave vector of the sample $[3,32]$. It is well-known that a climbing motion of the defect can be driven by an evolution of the whole pattern towards an optimal wave number [33]. A second source of climbing motion is the advection term in the order parameter equation due to mean flow and it is the form of the mean flow that we want to study in the following.

Considering the necessity of having a finite range of periodic solutions that are stable with respect to all secondary instabilities, we have chosen a large value for the nonlinear coefficient $\alpha$. As described by Rüdiger and Vinals [34] this causes a stabilization with respect to the zig-zag and the Eckhaus perturbations. Figure 3 shows the resulting various stability 


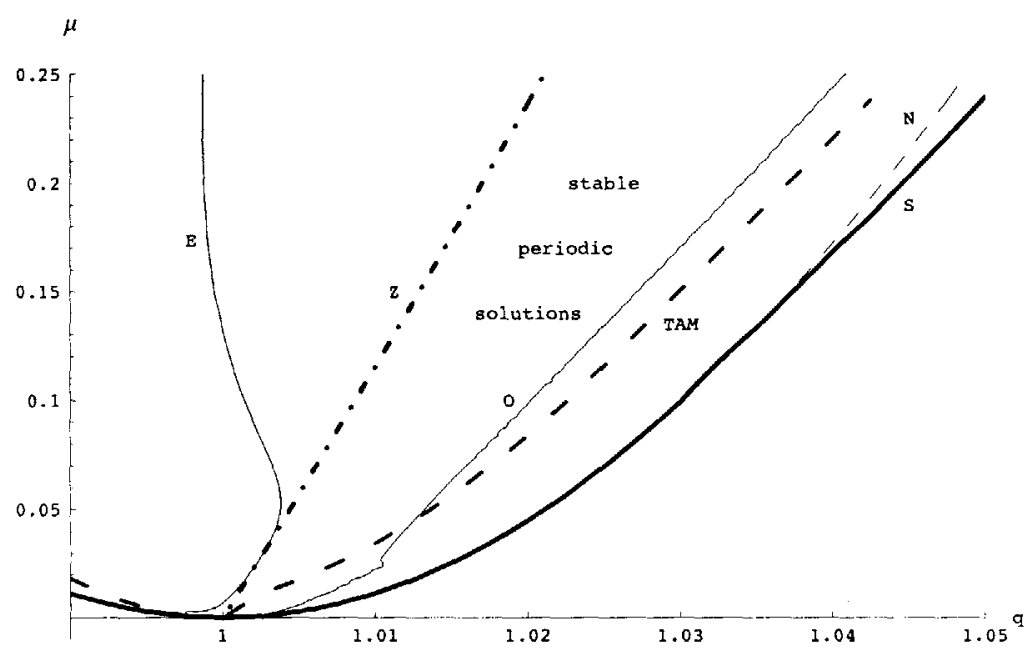

Fig. 3. Stability region for increased nonlinear damping $\alpha=0.5$ and $\beta_{1}=0.1$. Other values of the parameters used are $\gamma=0.1$, and $\Gamma=0.8$. We show the steady Eckhaus line (E), the oscillatory Eckhaus line (O), the zig-zag line (Z), and the TAM line (TAM). Periodic solutions exist above the neutral stability curve $(N)$ or, for $q>1$ the saddle node bifurcation (S). The region of stability of the basic solution against an Eckhaus instability is the region between the thin solid lines.

boundaries for the case $\beta_{1}=0.1$. The values used for the other parameters are $\gamma=0.1$ and $\Gamma=0.8$. The figure also includes the neutral stability curve of the basic periodic solution and, since the primary bifurcation is subcritical for $q>1$, we have included the saddle node curve where the periodic solution bifurcates. The range of basic solutions that is stable against all the perturbations considered here (Eckhaus, TAM, and zig-zag) is a region close to threshold at $\varepsilon=0$ between the zig-zag, TAM and oscillatory Eckhaus lines.

The parameters of Figure 3 are thus suitable for the numerical simulation of dislocations. In the simulations we have integrated Eqs. (27-31) and (34) on a two-dimensional domain of length $128 \pi$ (equivalent to about 60 stripes) using periodic boundary conditions in each direction [34]. The integration code exploits a Fourier function decomposition in a pseudo-spectral method with about 8 points per period of the basic Faraday pattern. A background pattern of wave number 1.0156 was used with two isolated dislocations placed in the sample thus creating a wave number of about 1.031 between the two defects. Although at the value $\varepsilon=0.15$ used for the simulations the second wave number is located in the unstable region, it 
turned out to be only slightly unstable and thus no secondary instability that could disturb the defect motion was detected at the time scale of the defect motion.

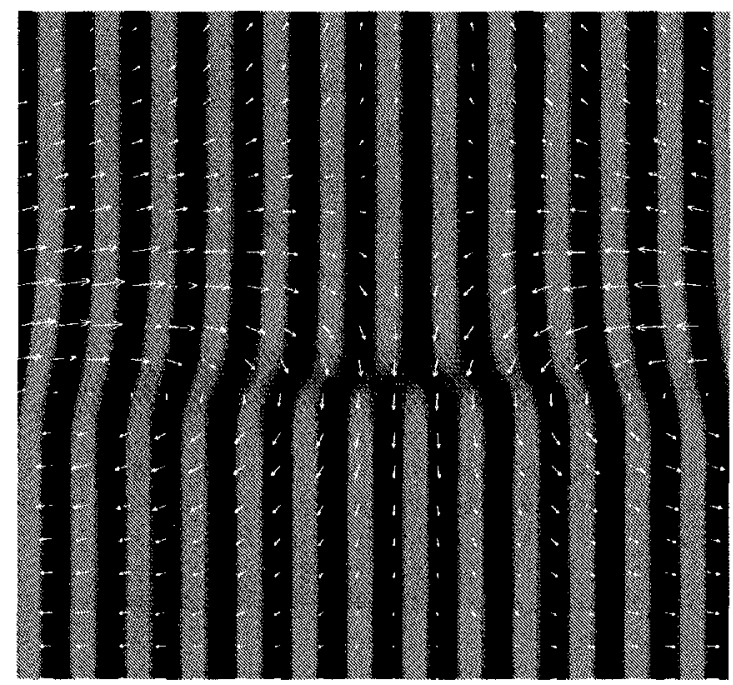

Fig. 4. The "ms" or long wave length component of the mean flow for $\beta_{1}=0.1$ and $\mu=$ 0.15 (other parameters as for Figure 3). The amplitude of the velocity is approximately 0.028 (maximal amplitude of the vertical component), and 0.013 (horizontal component). The defect moves downwards.

In the simulations the defects were generally found to be in a climbing motion with a velocity that converges to a steady value after short times. For vanishing mean flow $\left(\beta_{1}=0\right)$, as well as for all of the tested configurations with mean flow, the two defects move towards each other and annihilate, thus reducing the wave number of the pattern to 1.0156 . The velocity fields $U^{m s}$ as generated around a dislocation after a transient time are plotted in Figure 4. The amplitude of the small wave length part $U^{m o}$ is negligibly small. For the long wave length part $U^{m s}$ we found a pattern with two vortices close to each defect. The resulting flow at the defect drives the defect in the same direction as in comparable simulations for $\beta_{1}=0$ and therefore increases the velocity of the defect. The defect velocities for increasing $\beta_{1}$ are plotted in Figure 5 . 
The dominant advection flow generated by the defect is a long wavelength flow. This flow comprises two contributions: the viscous mean flow determined by Eq. (29) and the inviscid mean flow determined by Eq. (31). By increasing $\Gamma$ and thus suppressing the effect of the inviscid forcing term on $U^{m s}$ we have found a strongly decreasing component of the mean flow in direction of the climb. Therefore we conclude that at least for the current choice of parameters the main contribution to the advection of the defect originates from the inviscid forcing term in Eq. (31).

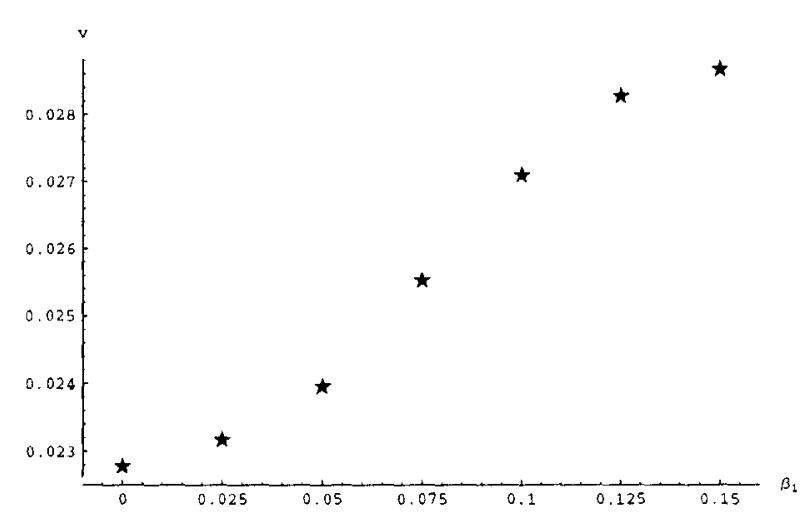

Fig. 5. Velocity of an isolated defect for $\mu=0.15$ and with increasing coupling to the mean flow (other parameters as for Figure 3).

\section{Conclusions}

We have reviewed recent work on the generation and action of mean flow for Faraday waves in a horizontally two-dimensional domain. As in similar hydrodynamical pattern forming systems, mean flow effects have been ignored in early studies, but, as has been shown in this article, they have important implications for the stability of patterns. In particular, mean flow couples to perturbations of the stripe pattern and thus changes the domain of stable regular solutions, generally in a destabilizing manner. The strongest coupling, and therefore the strongest destabilization occurs for longitudinal perturbations. More importantly, the mean flow generates a qualitatively new oscillatory longitudinal perturbation, which for small nonlinear damping in the phenomenological equation renders all stripe patterns unstable, resulting in time-dependent patterns at onset. A weaker effect of the mean 
flow contribution has been found for the TAM instability. It is interesting to note that the TAM perturbations, which are of finite wavelength, couple to the short-wave part of the mean flow, whereas the long wavelength Eckhaus perturbations couple to the large scale mean flow.

Furthermore we have presented preliminary results of numerical simulations of patterns with defects. A background pattern which is stable to secondary perturbations has been found by using large values of the nonlinear damping coefficient. Next, we studied the simplest case of a defect, a pair of dislocations moving in a pattern of perfect stripes. Using numerical simulations we found that the main flow generated by a dislocation is a large scale flow consisting of two vortices. In the parameter range considered here the mean flow increases the velocity of the dislocation. Simulations with different values of the gravity-capillary number $\Gamma$ showed that the main contribution to the climb of the dislocation originates from the inviscid forcing term.

Owing to the complexity of the derivation of mean flow equations we have limited the analysis to a specific limit in terms of the physical parameters. This concerns in particular the conditions on the dimensions of the layer. Further restrictions of the recent studies are the approximation of the surface waves using a phenomenological equation, and the assumption of a stripe pattern at onset. To consider more general situations and to test the results for the model equations described in this article further work needs to address these limitations.

\section{Acknowledgments}

This research was partially supported by the Spanish DGI under Grant BFM2001-2363 and by the U.S. Department of Energy under contract DEFG05-95ER14566. S.R. would like to thank J. Viñals for helpful discussions and critical comments.

\section{References}

(1) M. Faraday, Philos. Trans. R. Soc. London 121, 319 (1831).

[2] J. Miles and D. Henderson, Annu. Rev. Fluid Mech. 22, 143 (1990).

[3] M. Cross and P. Hohenberg, Rev. Mod. Phys. 65, 851 (1993).

[4] J. Gollub and J. Langer, Rev. Mod. Phys. 71, S396 (1999).

[5] A. Kudrolli and J. Gollub, Physica D 97, 133 (1996).

[6] D. Binks and W. van de Water, Phys. Rev. Lett. 78, 4043 (1997).

[7] D. Binks, M.-T. Westra, and W. van de Water, Phys. Rev. Lett. 79, 5010 (1997). 
[8] M.-T. Westra, D. Binks, and W. van de Water, J. Fluid Mech. 496, 1 (2003).

[9] S. Milner, J. Fluid Mech. 22581 (1991)

[10] W. Edwards and S. Fauve, J. Fluid Mech. 278, 123 (1994).

[11] W. Zhang, Ph.D. thesis, Florida State University (1994).

[12] W. Zhang and J. Viñals, J. Fluid Mech. 336, 301 (1997).

[13] J. M. Vega, E. Knobloch, and C. Martel, Physica D 154, 313 (2001).

[14] V. Lapuerta, C. Martel, and J. M. Vega, Physica D 173, 178 (2002).

[15] E. Knobloch, C. Martel, and J.M. Vega, Ann. N.Y. Acad. Sci. 974 (2002), 201-219

(16] E. Knobloch and J.M. Vega, In Geometry, Mechanics and Dynamics, Volume in honor of J.E. Marsden, P. Newton, P. Holmes, and A. Weinstein Eds, Springer-Verlag, (2002), 181.

[17] M. Higuera, J. M. Vega, and E. Knobloch, in Coherent Structures in Complex Systems, edited by L. Bonilla, G. Platero, D. Reguera, and J. Rubi (Springer-Verlag, New York, 2001), p. 328.

[18] M. Higuera, J. M. Vega, and E. Knobloch, J. Nonlinear Sci. 12, 505 (2002).

[19] J. M. Vega, S. Rüdiger, and J. Viñals, to be published.

[20] A. Davey and S. Stewartson, Proc. R. Soc. London A 338, 101 (1974).

[21] M. Longuet-Higgins, Phil. Trans. Roy. Soc. A 245, 535 (1953).

[22] J. Swift and P. Hohenberg, Phys. Rev. A 15, 319 (1977).

[23] W. Zhang and J. Viñals, Phys. Rev. Lett. 74, 690 (1995).

[24] V. Zakharov, Zh. Prikl. Mekh. Tekh. Fiz. 9, 86 (1968), [J. Appl. Mech. Tech. Phys. 9, 190 (1968)].

[25] D. Crawford, P. Saffman, and H. Yuen, Wave Motion 2, 1 (1980).

[26] L. Landau and E. Lifshitz, Mechanics, Pergamon, New York, 1976.

[27] P. Lyngshansen and P. Alstrom, J. Fluid Mech. 351, 301 (1997).

[28] P. Chen and J. Viñals, Phys. Rev. Lett. 79, 2670 (1997).

[29] W. Zhang and J. Viñals, unpublished.

[30] J. Nicolás and J. M. Vega, Fluid Dyn. Research 32, 119 (2003).

[31] P. Chen and K. Wu, Phys. Rev. Lett. 85, 3813 (2000).

[32] A. C. Newell, T. Passot, and J. Lega, Annu. Rev. Fluid Mech. 25, 399 (1993).

[33] T. Walter, W. Pesch, and E. Bodenschatz, to be published.

[34] S. Rüdiger and J. Viñals, to be published. 